\title{
Matrix metalloproteinase type 2 expression in malignant adrenocortical tumors: diagnostic and prognostic significance in a series of 50 adrenocortical carcinomas
}

\author{
Marco Volante ${ }^{1}$, Paola Sperone ${ }^{2}$, Enrico Bollito ${ }^{1}$, Elena Frangipane ${ }^{1}$, Rosj Rosas ${ }^{1}$, \\ Fulvia Daffara ${ }^{3}$, Massimo Terzolo ${ }^{3}$, Alfredo Berruti ${ }^{2}$ and Mauro Papotti ${ }^{3}$
}

${ }^{1}$ Division of Pathology, Department of Clinical \& Biological Sciences, University of Turin and San Luigi Hospital, Orbassano, Torino, Italy; ${ }^{2}$ Division of Oncology, Department of Clinical \& Biological Sciences, University of Turin and San Luigi Hospital, Orbassano, Torino, Italy and ${ }^{3}$ Division of Internal Medicine, Department of Clinical \& Biological Sciences, University of Turin and San Luigi Hospital, Orbassano,

Torino, Italy

\begin{abstract}
The differential diagnosis of adrenocortical carcinoma from adrenocortical adenoma is based on different pathological parameters, usually incorporated in scoring systems, which unfortunately lack a $100 \%$ sensitivity and specificity. Little is known on the molecular mechanisms leading to the malignant phenotype in adrenocortical tumors. Among other molecules, metalloproteinases were demonstrated to be implicated in malignant progression and metastatization of solid tumors, including endocrine ones. Therefore, we aimed to investigate metalloproteinases and their inhibitors expression in a series of 50 adrenocortical carcinomas and 50 control adrenocortical adenomas, diagnosed according to the Weiss histological criteria. Immunohistochemical results were scored by semiquantitative analysis and compared with clinicopathological parameters and outcome. Metalloproteinase type 2 gave the most significant result, being detected in neoplastic cells in $1 / 50$ adrenocortical adenomas $(2 \%)$ and $37 / 50$ adrenocortical carcinomas $(74 \%)(P<0.001)$, with a focal (score $1,<20 \%$ of positive cells-two-thirds of cases) or diffuse (score $2,>20 \%$ of positive cells-one-third of cases) pattern. In addition, diffuse (score 2) metalloproteinase type 2 protein expression, as compared to focal or negative immunostaining, correlated with shorter survival $(P<0.02)$ and disease-free interval $(P=0.05)$. No correlation was found comparing metalloproteinase type 2 expression and any clinicopathological parameter. Our data indicate that metalloproteinase type 2 immunohistochemical localization in tumor cells is significantly restricted to malignant adrenocortical tumors, with high specificity but low sensitivity. In addition, a strong metalloproteinase type 2 expression in adrenocortical carcinoma was for the first time recognized as an unfavorable prognostic factor.

Modern Pathology (2006) 19, 1563-1569. doi:10.1038/modpathol.3800683; published online 15 September 2006
\end{abstract}

Keywords: adrenal cortex; carcinoma; differential diagnosis; immunohistochemistry; MMP-2

Adrenocortical carcinoma is a rare tumor of the adrenal cortex, which accounts for no more than $0.2 \%$ of all malignancies. Its incidence is approximately one per million with females affected more frequently than males. Two peaks of incidence are observed in early childhood and adults over age of 60

Correspondence: Dr M Volante, MD, Division of Pathology, Department of Clinical and Biological Sciences, University of Turin and San Luigi Hospital, Regione Gonzole 10, Orbassano, Torino I-10043, Italy.

E-mail: marco.volante@unito.it

Received 30 May 2006; revised 27 July 2006; accepted 31 July 2006; published online 15 September 2006 years. Up to two-thirds of cases have distant metastases at the time of diagnosis and the mortality exceeds $90 \%$ with a mean survival of less than 30 months. ${ }^{1-3}$

The differential diagnosis of carcinomas from adenomas has been classically based on several pathological and clinical parameters: indeed, taken alone, none of these is indicative of malignancy (with the possible exception of clear-cut vascular invasion and atypical mitoses), but the combination of some of them may lead to a correct diagnosis of carcinoma. Different scoring systems for adrenocortical carcinoma have been proposed, based on statistical evidence of malignant behavior in tumors reaching a given threshold of histomorphological 
parameter expression. ${ }^{2,4-6}$ Unfortunately, such scoring systems are sophisticated, time consuming, difficult to apply and not easily reproducible. ${ }^{1,7,8}$ In addition, although the majority of adrenocortical carcinomas are associated with typical features of malignancy such as necrosis, hemorrhage, vascular invasion, high mitotic count and do not really require a numerical scoring of the various parameters for a final diagnosis of cancer, there are several other cases in which the distinction from their benign counterparts is not straightforward. These cases are a challenge for the pathologist, as the therapeutic strategy in adrenocortical carcinomas is radically different from that of adenomas and an accurate diagnosis is mandatory.

To this purpose, the classical scoring systems have been improved and revisited, with the aim of providing an easier system of evaluation, ${ }^{1}$ and the individual importance and the diagnostic impact of the various parameters classically considered was re-assessed..$^{9}$ Finally, another approach was to combine the morphological analysis with the identification of markers of malignancy. Among these, ploidy, ${ }^{10}$ cytogenetic or oncogene analysis of p16 and p53 were proposed as differential markers of adenomas from carcinomas. ${ }^{11,12}$ The proliferative activity was also considered useful to discriminate adenomas from carcinomas, with a cutoff of Ki-67 index varying from 2.5 to $4 \%$, according to the different authors. ${ }^{1,13,14}$ Other less common markers tested in adrenocortical carcinoma include insulin growth factors (IGF-1 and IGF-2), vascular endothelial growth factor (VEGF), gelatinase, membrane type-1 matrix metalloproteinase (MMP) and others. ${ }^{15-17}$ Among these, by in situ hybridization, gelatinase A (MMP type 2 (MMP-2)) mRNA was found to be overexpressed in stromal cells (but not in tumor cells) of 13/16 adrenocortical carcinomas and in only one of 14 adenomas. ${ }^{16}$ Conversely, serum levels of this molecule were not significantly different in the two tumor types (whereas VEGF and MMP-3 were significantly higher in adrenocortical carcinoma patients) according to another study. ${ }^{17}$

In order to clarify the presence and possible diagnostic implications of MMPs and their inhibi- tors in adrenocortical tumors, an immunohistochemical study was designed in a large series of 50 adrenocortical carcinomas and in a control group of 50 adrenocortical adenomas. We here show a peculiar MMP-2 protein expression in malignant adrenocortical tumors, which may be a useful adjunct for the diagnosis of adrenocortical carcinoma, and may also bear adverse prognostic significance.

\section{Materials and methods}

\section{Tumor Samples Selection}

Fifty cases with a diagnosis of adrenocortical carcinomas were collected from the clinical databases of the Divisions of Oncology and Endocrinology at San Luigi Hospital, University of Turin, that serves as referral center for this rare neoplasm. For all patients, pathological material, including original histological slides and paraffin blocks, were retrieved from the files of the Pathology Departments of the University of Turin at Molinette (26 cases) and San Luigi Hospitals (24 cases, including 16 consultation cases, operated elsewhere). From the same institutions, a control group of 50 adrenocortical adenomas operated in the same period was also collected. A thorough histopathological review of all cases, including re-assessment of the commonly used Weiss system, was performed independently by three of us (MV, EB, MP). For comparison, all cases were also scored using the Weiss modified (according to Aubert et $a l^{1}$ ) and Van Slooten scoring systems. For all cases, complete follow-up information were available for survival analysis. The main clinicopathological features of the two groups are reported in Table 1. The study was approved by the Institutional Review Board of the hospital.

\section{Methods}

Serial $5-\mu \mathrm{m}$-thick paraffin sections were collected onto charged slides and processed by immunohistochemistry in a pilot series of 10 cases of

Table 1 Clinicopathological features of a series of 100 adrenocortical tumors

\begin{tabular}{|c|c|c|c|c|c|c|}
\hline Diagnosis (\#) & $F / M$ & $\begin{array}{l}\text { Age mean } \\
\text { (range) }\end{array}$ & Location (\%) & $\begin{array}{c}\text { Size (cm) } \\
\text { mean (range) }\end{array}$ & $\begin{array}{l}\text { Weight }(\mathrm{g}) \text { mean } \\
\quad \text { (range) }\end{array}$ & Functional status (\%) \\
\hline $\begin{array}{l}\text { Adrenocortical } \\
\text { carcinoma (50) }\end{array}$ & 1.4 & $46(20-85)$ & $\begin{array}{l}\text { LA } 51 \\
\text { RA } 49\end{array}$ & $11.1(3-25)$ & $257(30-1630)$ & $\begin{array}{l}\text { NF (54) } \\
\text { Cortisol (20) } \\
\text { Aldosterone (6) } \\
\text { Androgens (16) } \\
\text { Not known (4) }\end{array}$ \\
\hline $\begin{array}{l}\text { Adrenocortical } \\
\text { adenoma (50) }\end{array}$ & 2.3 & $52.3(15-77)$ & $\begin{array}{l}\text { LA } 51 \\
\text { RA } 49\end{array}$ & $3.4(1-10)$ & $33.6(0.8-150)$ & $\begin{array}{l}\text { NF (26) } \\
\text { Cortisol (40) } \\
\text { Aldosterone (31) } \\
\text { Not known (3) }\end{array}$ \\
\hline
\end{tabular}

LA: left adrenal; RA: right adrenal; NF: not functioning. 
adrenocortical carcinomas and 10 adrenocortical adenomas with the following primary antibodies, which recognize a set of markers mostly involved in angiogenetic and metastatic processes: cathepsin $\mathrm{K}$ (Novocastra, Newcastle, UK), VEGF and MMP-2 (NeoMarkers/LabVision, Fremont, CA, USA), MMP9, TIMP-1 and TIMP-2 (LabVision, Fremont, CA, USA). A standard automated (Dako Autostainer, Glostrup, Denmark) immunoperoxidase procedure was employed, and the appropriate working conditions were set in the laboratory by testing appropriate internal and external controls in parallel. Detailed working conditions for all antibodies used in the study are reported in Table 2. Immunoreactions were revealed by a biotin-free dextran-chain detection system (Envision, Dako), and developed using diaminobenzidine as the chromogen. The specificity of the antibodies was validated in parallel negative control sections by omitting the primary antibodies for each immunohistochemical run

For all markers, the immune reaction was assessed by a semiquantitative score according to the percentage of positive tumor cells (score 0-2: $0 \%$, less than or equal to $20 \%$ and more than $20 \%$, respectively).

Significant preliminary results were obtained for MMP-2 only; therefore, the immunohistochemical profile was assessed in the whole series of 100 cases for that marker, only.

\section{Statistical Analysis}

All data were analyzed with STATISTICA for Windows software version 6.1 (Stat Soft, Italy). A level of $P<0.05$ was considered statistically significant. The differential expression of the markers in the carcinoma and adenoma groups, as well as correlation between MMP-2 expression and clinicopathological parameters, was estimated by chisquare test, chi-square for trend and Kruskal-Wallis analysis of variance for non-parametric data, when indicated. Univariate survival analysis was based on the Kaplan-Meier product limit estimate of overall survival distribution. Unadjusted differences between survival curves were tested using the log-rank test.

\section{Results}

The preliminary immunohistochemical results in the pilot series of 20 adrenal tumors identified the selective presence of MMP-2 immunostaining in adrenocortical carcinoma. All other markers tested were either completely negative in both adenomas and carcinomas, as for cathepsin $\mathrm{K}$, or heterogeneously expressed in both groups (as well as in peritumoral adrenal cortex), as for VEGF, TIMP-1 and 2 and MMP-9. Based on these findings, we analyzed the MMP-2 expression profile in all 50 adrenocortical carcinomas and 50 benign control adrenocortical adenoma samples, finding a strong correlation between MMP-2 protein expression in tumor cells and malignant phenotype in adrenocortical tumors. MMP-2 protein was detected in the neoplastic cells of $37 / 50$ carcinomas $(74 \%)$, either focally (score 1, less than or equal to $20 \%$ of tumor cells) in 26 cases (approximately two-thirds of positive cases), or diffusely (score 2, more than $20 \%$ of tumor cells) in 11 cases (one-third of positive cases). The immunohistochemical signal had a moderate to strong intensity and was diffuse in the cytoplasm of tumor cells (Figure 1). In contrast, all but one case of adrenocortical adenoma were unreactive (as compared to carcinomas, $P<0.001$, chi-square 51.995). Interestingly, the single MMP-2-positive adenoma, which presented a focal reactivity in scattered tumor cells (score 1), was classified as benign based on a Weiss score 2, but had a Van Slooten score of 9.8 (ie in the range of malignancy) and borderline clinicopathological parameters including suspected venous invasion with predominant eosinophilic cells, and a borderline mitotic index (four mitoses $\times 50$ high power fields), as well as a large size $(8 \mathrm{~cm})$ and a weight of $135 \mathrm{~g}$.

Table 2 List of antibodies tested

\begin{tabular}{|c|c|c|c|c|c|c|}
\hline Antibody & Source & Type & Clone & Dilution & Antigen retrieval & Incubation \\
\hline Cathepsin K & Novocastra (Newcastle, UK) & Mono & CK4 & $1: 100$ & $\begin{array}{l}\text { MW CT buffer (pH 6) } \\
35 \text {-min cycles }\end{array}$ & $40 \mathrm{~min} \mathrm{RT}$ \\
\hline VEGF & NeoMarkers (Fremont, CA, USA) & Poly & - & $1: 250$ & $\begin{array}{l}\text { MW EDTA buffer (pH 8) } \\
35 \text {-min cycles }\end{array}$ & Overnight $4^{\circ} \mathrm{C}$ \\
\hline MMP-2 & NeoMarkers (Fremont, CA, USA) & Mono & A-Gel-VC2 & $1: 100$ & None & 40 min $\mathrm{RT}$ \\
\hline MMP-9 & Lab Vision (Fremont, CA, USA) & Poly & - & $1: 150$ & $\begin{array}{l}\text { MW CT buffer (pH 6) } \\
35 \text {-min cycles }\end{array}$ & Overnight $4^{\circ} \mathrm{C}$ \\
\hline TIMP-1 & Lab Vision (Fremont, CA, USA) & Poly & - & $1: 100$ & $\begin{array}{l}\text { MW CT buffer (pH 6) } \\
35 \text {-min cycles }\end{array}$ & $40 \mathrm{~min} \mathrm{RT}$ \\
\hline TIMP-2 & Lab Vision (Fremont, CA, USA) & Poly & - & $1: 100$ & $\begin{array}{l}\text { MW CT buffer (pH 6) } \\
35 \text {-min cycles }\end{array}$ & Overnight $4^{\circ} \mathrm{C}$ \\
\hline
\end{tabular}

mono: monoclonal; poly: polyclonal; RT: room temperature; MW: microwave; CT: citrate. 

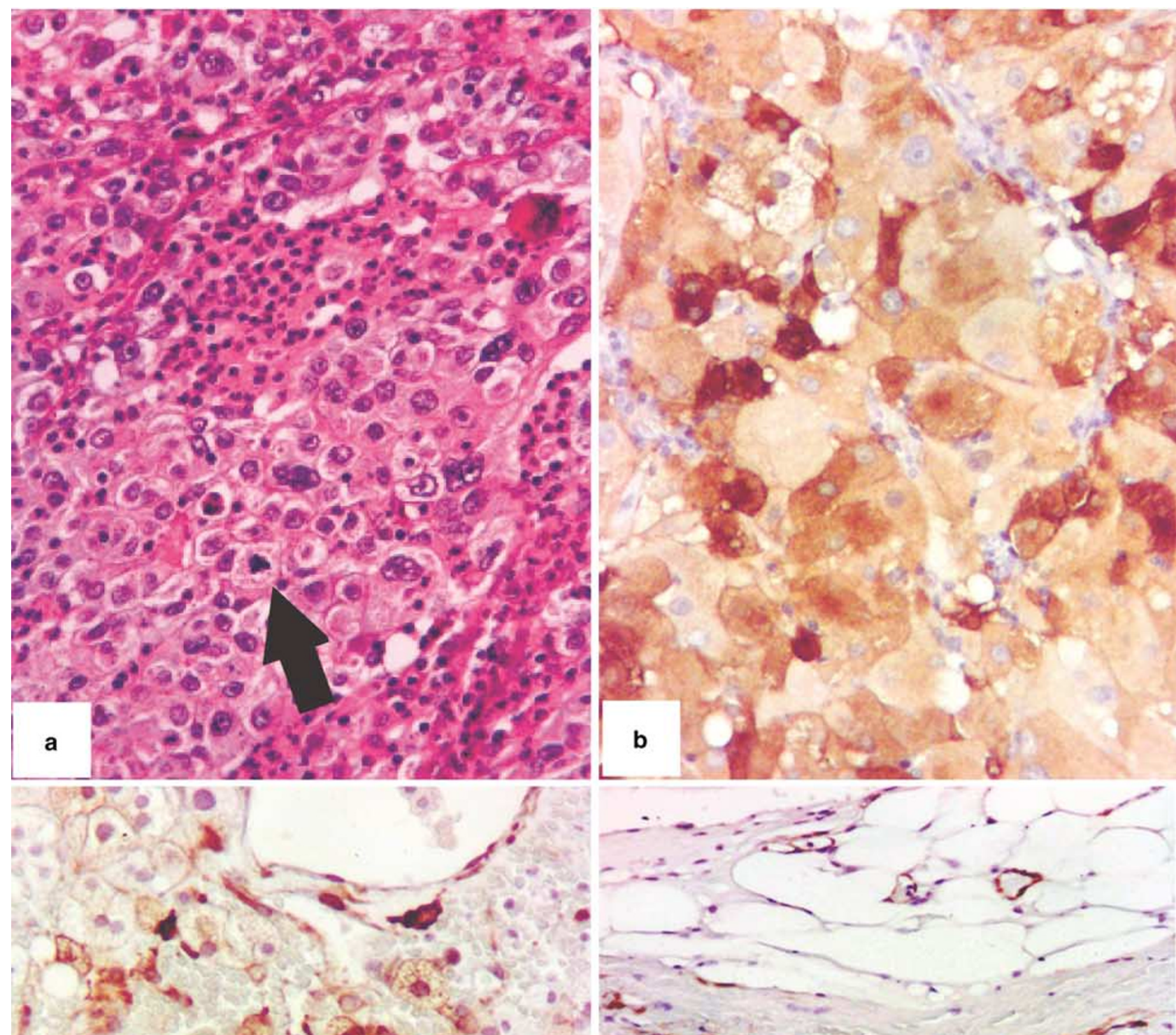

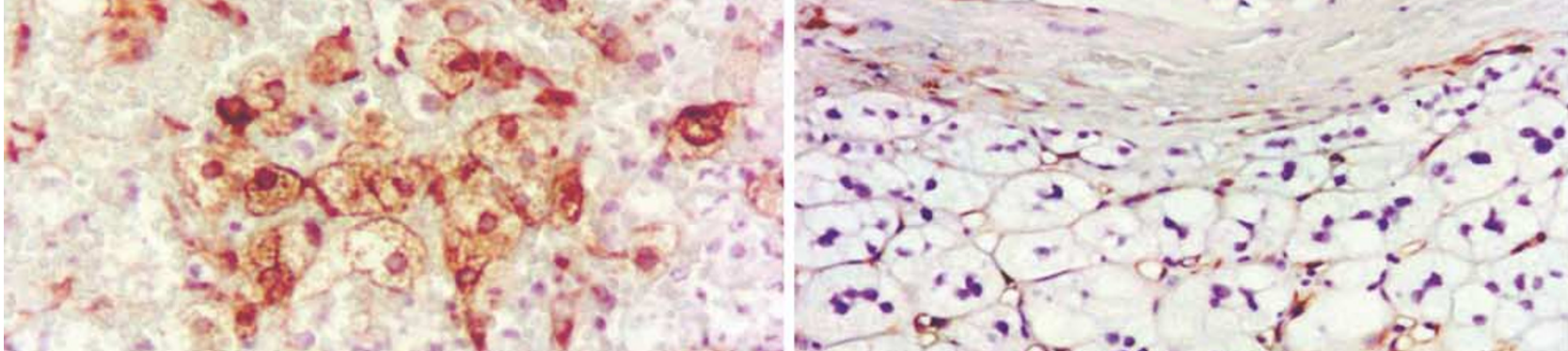

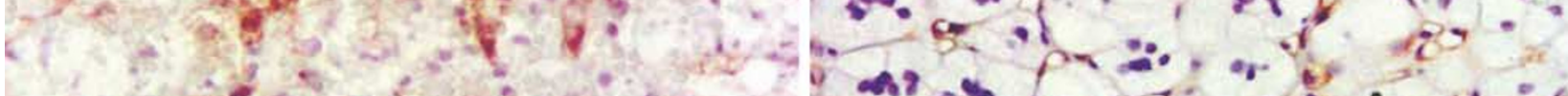

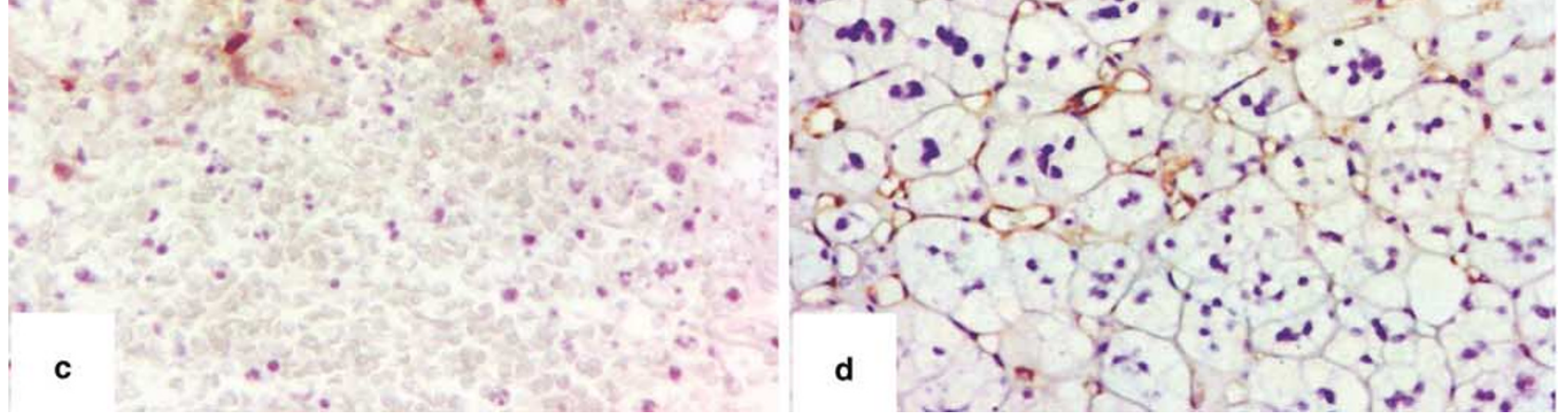


In two cases, tissue from the primary carcinomas and the corresponding lung metastases were available for comparison: a partially similar MMP-2 immunohistochemical pattern was observed, with an increased expression (from score 1 to score 2) in the lung sample of one case.

Concerning non-neoplastic cells, constitutive MMP-2 expression was found in vascular endothelia of both surrounding normal adrenal tissue and the intra-tumoral vascular network (Figure 1d), and, to a minor extent, in elongated stromal cells of the capsule of both benign and malignant tumors.

No correlation between MMP-2 immunohistochemical expression and any clinicopathological parameter tested (sex, age, size, weight, hormonal secretion, Weiss score and each individual criteria, Van Slooten score) could be determined. Interestingly, MMP-2 protein expression was detected in five of the seven adrenocortical carcinoma cases with a size smaller than $5 \mathrm{~cm}$, which may represent a particular problem for the differential diagnosis with adrenocortical adenoma. In the same way, the 13 MMP-2-negative cases lacked peculiar pathological features.

Conversely, a statistically significant prognostic role of MMP-2 expression in adrenocortical carcinomas was identified. Strong MMP-2 protein expression (score 2), as opposed to negative or focal (score 1) MMP-2 expression, had a significant impact on poor outcome, in terms of both overall survival $(P<0.02)$, and shorter disease-free interval $(P=0.05)$. A median overall survival of 74.8 months (range 2-346 months) was observed for all cases, and of 31 months (range 3.3-97.4 months) in cases with score 2 MMP-2 immunoreactivity, whereas was not reached for cases with absent or score 1 MMP-2 immunoreactivity (Figure 2).

\section{Discussion}

In the present study, a useful diagnostic role of MMP-2 immunohistochemical analysis in the identification of malignant adrenocortical tumors was observed, MMP-2 protein expression being restricted to neoplastic cells in a high percentage of carcinoma cases.

This is a valid adjunct to the histological diagnosis of adrenocortical carcinoma, which is based on the recognition of morphological characteristics related to malignancy, either considered alone or combined in scoring systems. Although most cases of adrenocortical carcinomas may present unequivocal features of malignancy, some cases may show questionable carcinoma features, and their correct histopathological definition may be problematic.

Our interest in MMPs as indicators of malignant phenotype in adrenocortical tumors, stemmed from the fact that MMPs are a family of endopeptidases capable of degrading all components of the extracellular matrix, which play a pivotal role in morphogenesis, physiological and pathological conditions. ${ }^{18}$ Such family consists of at least 18 structurally related members classified according to their primary structure and substrate specificity, which possess a proteolytic domain, whose activity is selectively inhibited by specific ubiquitarious tissue inhibitors (TIMPs). The role of MMPs, and
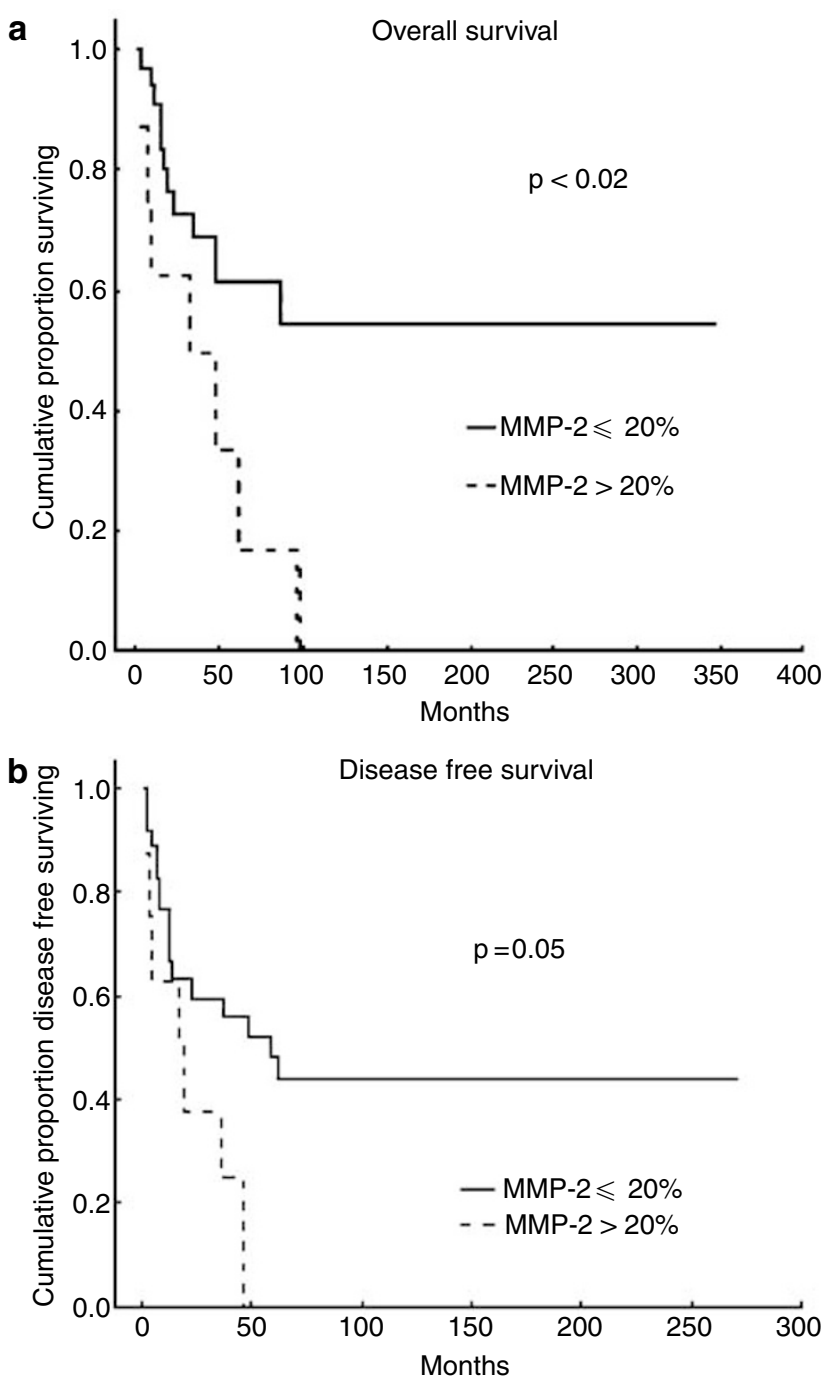

Figure 2 Overall survival distribution (a) and disease-free survival distribution (b) in adrenocortical carcinomas, as compared to MMP2 protein expression.

Figure 1 MMP2 protein expression in adrenocortical carcinoma with nuclear pleomorphism and atypical mitoses (a, arrow). The immunohistochemical reactivity was either diffuse (b) or focal (c). A control adrenocortical adenoma had no MMP2 immunoreactivity in neoplastic cells, although the extracapsular and intratumoral vascular endothelial network was constitutively expressing MMP2 (d) (a: H\&E; b, c, d: immunoperoxidase; a, d: $\times 20 ; \mathbf{b}, \mathbf{c}: \times 40)$. 
particularly of gelatinases (MMP-2 and MMP-9), in neoplastic progression has been postulated on the base of in vitro and in vivo effects on degrading basement membrane and extracellular matrix and by promoting tumor growth. ${ }^{19,20}$ Numerous studies in a variety of solid tumors, including lung, colon, pancreas and breast cancers, demonstrated a production of MMP by neoplastic cells and correlated their presence with poor clinical outcome. ${ }^{21-28} \mathrm{In}$ endocrine tumors, MMP-2 has been investigated mostly in pituitary ${ }^{29}$ and thyroid $^{30}$ tumors. A report $^{16}$ on MMP-2 (gelatinase A) expression in adrenocortical tumors, as detected by in situ hybridization, claimed the presence of RNA transcripts in peritumoral stromal cells in malignant tumors as compared to benign lesions. In contrast, no evidence of MMP-2 expression in neoplastic cells was reported. The presence of MMP-2 expression in stromal cells, including fibroblasts, inflammatory cells and vascular endothelia, has been well documented $^{31}$ even in our present experience, and supports the findings from Kjellman et al. ${ }^{16} \mathrm{How}^{-}$ ever, in our study MMP-2 protein was also specifically detected in neoplastic cells of malignant lesions, but not of adenomas (which expressed MMP-2 in the vascular network only). In a pilot study using real-time PCR, we found, among several other molecules investigated, MMP-2 mRNA expression being 20 times higher in a case of adrenocortical carcinoma as compared to a case of adrenocortical adenoma (data not shown); however, as such highly sensitive method is not able to localize the specific target transcripts, and nonneoplastic sources of MMP-2 (such as blood vessels) are widely represented also in normal and benign adrenal tissue, an immunohistochemical approach was preferred.

As opposed to MMP-2, other members of MMPs family, such as the other gelatinase MMP-9, or their inhibitors (TIMP-1 and 2), as well as other angiogenetic (VEGF) or enzymatic (cathepsin K) factors failed to show a specific distribution pattern among adrenocortical lesions, therefore restricting to MMP2 the diagnostic role as a marker of malignancy in adrenocortical carcinomas.

Interestingly, MMP-2 expression in adrenocortical carcinoma was not related to the Weiss score number of individual tumors. In other terms, MMP-2 protein expression was not limited to high Weiss score clear-cut carcinomas, but may also be a useful adjunct (with a low sensitivity of $74 \%$, but a very high specificity of $98 \%$ ) to support a diagnosis of carcinoma in those lesions with a Weiss score just above the cutoff level and in the lower range of malignancy, which generally cause the most relevant diagnostic difficulties. In contrast, the presence of a focal pattern (less than or equal to $20 \%$ of positive tumor cells) in the majority of the positive malignant cases could limit the applicability of MMP-2 as a diagnostic marker in core biopsies of adrenal masses.
Moreover, as MMP-2 functions are related to extracellular matrix degradation, a selective association of MMP-2 expression with specific pathological features (ie necrosis and/or vascular invasion) could be expected; however, none of the parameters included in the Weiss scoring system individually correlated to MMP-2 protein expression in the statistical analysis.

Apart from its diagnostic utility, strong (score 2) MMP-2 immunohistochemical expression was related to a more aggressive behavior in terms of outcome and disease-free interval, and may therefore be considered a predictive factor of unfavorable clinical evolution of adrenocortical carcinoma, for which definite histopathological prognostic factors have not been fully established. ${ }^{7}$

Moreover, although in vivo models are necessary to clarify the molecular regulation of MMP-2 in adrenocortical carcinoma, a potential clinical implication of MMP-2 protein distribution analysis in adrenocortical tumor cells is represented by the very recent characterization of specific peptide or nonpeptide MMP inhibitors, which may be used in radioimaging techniques, ${ }^{32}$ as well as therapeutic agents. ${ }^{19}$

In conclusion, we have shown that MMP-2 immunohistochemical staining is an easy and helpful tool to confirm the diagnosis of adrenal carcinoma, with a low sensitivity but a very high specificity. Classical methods based on a combination of morphological parameters assessed in a scoring system may gain benefit from the addition and/or replacement of immunohistochemical markers in the scoring. In this respect, the combined use of MMP-2 in a panel of morphological and other immunohistochemical (ie Ki-67) markers may further increase the accuracy of the diagnosis of adrenocortical carcinoma. In addition, as no clinical or pathological parameter is to date predictive of the clinical evolution in malignant adrenocortical lesions, MMP-2 protein expression may help to segregate those cases with the worst prognosis.

\section{Acknowledgements}

We are indebted to Professor G Bussolati (University of Turin) for his suggestions and for providing access to part of the histological material. Work supported by grants from the Italian Ministry of University and Research (MIUR), Rome (ex 60\% to MV, MT and MP, and PRIN-ex 40\%-number 2005067583_005).

\section{References}

1 Aubert S, Wacrenier A, Leroy X, et al. Weiss system revisited: a clinicopathologic and immunohistochemical study of 49 adrenocortical tumors. Am J Surg Pathol 2002;26:1612-1619. 
2 DeLellis RA, Lloyd RV, Heitz PU (eds). World Health Organization Classification of Tumors, Pathology and Genetics, Tumors of Endocrine Organs. Lyon, France: IARC Press, 2004.

3 LiVolsi VA, Asa SL. Endocrine Pathology. Philadelphia, PA: Churchill Livingstone, 2002.

4 Hough AJ, Hollifield JW, Page DL, et al. Prognostic factors in adrenal cortical tumors. A mathematical analysis of clinical and morphologic data. Am J Clin Pathol 1979;72:390-399.

5 van Slooten H, Schaberg A, Smeenk D, et al. Morphologic characteristics of benign and malignant adrenocortical tumors. Cancer 1985;55:766-773.

6 Weiss LM, Medeiros LJ, Vickery Jr AL. Pathologic features of prognostic significance in adrenocortical carcinoma. Am J Surg Pathol 1989;13:202-206.

7 Aiba M, Fujibayashi M. Histopathological diagnosis and prognostic factors in adrenocortical carcinoma. Endocr Pathol 2005;16:13-22.

8 Wieneke JA, Thompson LD, Heffess CS. Adrenal cortical neoplasms in the pediatric population: a clinicopathologic and immunophenotypic analysis of 83 patients. Am J Surg Pathol 2003;27:867-881.

9 Harrison LE, Gaudin PB, Brennan MF. Pathologic features of prognostic significance for adrenocortical carcinoma after curative resection. Arch Surg 1999;134:181-185.

10 Medeiros LJ, Weiss LM. New developments in the pathologic diagnosis of adrenal cortical neoplasms. A review. Am J Clin Pathol 1992;97:73-83.

11 Pilon C, Pistorello M, Moscon A, et al. Inactivation of the p16 tumor suppressor gene in adrenocortical tumors. J Clin Endocrinol Metab 1999;84:2776-2779.

12 Pinto EM, Billerbeck AE, Fragoso MC, et al. Deletion mapping of chromosome 17 in benign and malignant adrenocortical tumors associated with the Arg337His mutation of the p53 tumor suppressor protein. J Clin Endocrinol Metab 2005;90:2976-2981.

13 Stojadinovic A, Ghossein RA, Hoos A, et al. Adrenocortical carcinoma: clinical, morphologic, and molecular characterization. J Clin Oncol 2002;20:941-950.

14 Terzolo M, Boccuzzi A, Bovio S, et al. Immunohistochemical assessment of Ki-67 in the differential diagnosis of adrenocortical tumors. Urology 2001;57: 176-182.

15 Fottner Ch, Hoeflich A, Wolf E, et al. Role of the insulin-like growth factor system in adrenocortical growth control and carcinogenesis. Horm Metab Res 2004;36:397-405.

16 Kjellman M, Enberg U, Hoog A, et al. Gelatinase A and membrane-type 1 matrix metalloproteinase mRNA: expressed in adrenocortical cancers but not in adenomas. World J Surg 1999;23:237-242.

17 Kolomecki K, Stepien H, Bartos M, et al. Usefulness of VEGF, MMP-2, MMP-3 and TIMP-2 serum level evaluation in patients with adrenal tumours. Endocr Regul 2001;35:9-16.
18 Chambers AF, Matrisian LM. Changing views of the role of matrix metalloproteinases in metastasis. J Natl Cancer Inst 1997;89:1260-1270.

19 Hidalgo M, Eckhardt SG. Development of matrix metalloproteinase inhibitors in cancer therapy. J Natl Cancer Inst 2001;93:178-193.

20 Mira E, Manes S, Lacalle RA, et al. Insulin-like growth factor I-triggered cell migration and invasion are mediated by matrix metalloproteinase-9. Endocrinology 1999;140:1657-1664.

21 Bramhall SR. The matrix metalloproteinases and their inhibitors in pancreatic cancer. From molecular science to a clinical application. Int J Pancreatol 1997;21:1-12.

22 Curran S, Dundas SR, Buxton J, et al. Matrix metalloproteinase/tissue inhibitors of matrix metalloproteinase phenotype identifies poor prognosis colorectal cancers. Clin Cancer Res 2004;10:8229-8234.

23 Davidson B, Goldberg I, Liokumovich P, et al. Expression of metalloproteinases and their inhibitors in adenocarcinoma of the uterine cervix. Int J Gynecol Pathol 1998;17:295-301.

24 Hashimoto K, Kihira Y, Matuo Y, et al. Expression of matrix metalloproteinase-7 and tissue inhibitor of metalloproteinase-1 in human prostate. J Urol 1998; 160:1872-1876.

25 Kallakury BV, Karikehalli S, Haholu A, et al. Increased expression of matrix metalloproteinases 2 and 9 and tissue inhibitors of metalloproteinases 1 and 2 correlate with poor prognostic variables in renal cell carcinoma. Clin Cancer Res 2001;7:3113-3119.

26 Kugler A, Hemmerlein B, Thelen P, et al. Expression of metalloproteinase 2 and 9 and their inhibitors in renal cell carcinoma. J Urol 1998;160:1914-1918.

27 Nawrocki B, Polette M, Marchand V, et al. Expression of matrix metalloproteinases and their inhibitors in human bronchopulmonary carcinomas: quantificative and morphological analyses. Int J Cancer 1997;72:556-564.

28 Sutinen M, Kainulainen T, Hurskainen $\mathrm{T}$, et al. Expression of matrix metalloproteinases (MMP-1 and -2) and their inhibitors (TIMP-1, -2 and -3) in oral lichen planus, dysplasia, squamous cell carcinoma and lymph node metastasis. Br J Cancer 1998;77: 2239-2245.

29 Paez Pereda M, Ledda MF, Goldberg V, et al. High levels of matrix metalloproteinases regulate proliferation and hormone secretion in pituitary cells. J Clin Endocrinol Metab 2000;85:263-269.

30 Campo E, Merino MJ, Liotta L, et al. Distribution of the 72-kd type IV collagenase in nonneoplastic and neoplastic thyroid tissue. Hum Pathol 1992;23:1395-1401.

31 Dano K, Romer J, Nielsen BS, et al. Cancer invasion and tissue remodeling-cooperation of protease systems and cell types. APMIS 1999;107:120-127.

32 Medina OP, Kairemo K, Valtanen H, et al. Radionuclide imaging of tumor xenografts in mice using a gelatinasetargeting peptide. Anticancer Res 2005;25:33-42. 